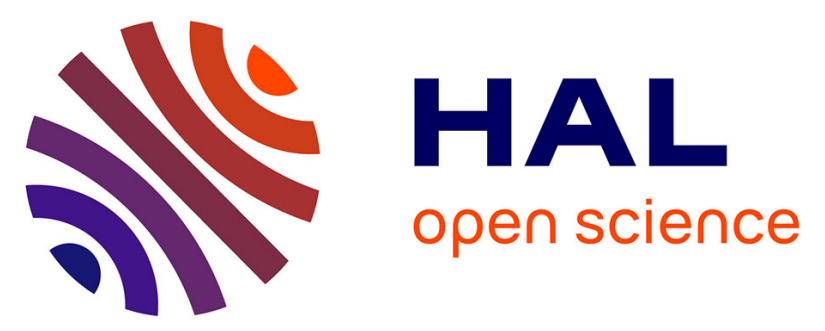

\title{
High enthalpy source dedicated to quantitative infrared emission spectroscopy of gas flows at elevated temperatures
}

Robert Georges, Jonathan Thiévin, Abdessamad Benidar, Sophie Carles, B. Amyay, M. Louviot, Vincent Boudon, J. Vander Auwera

\section{To cite this version:}

Robert Georges, Jonathan Thiévin, Abdessamad Benidar, Sophie Carles, B. Amyay, et al.. High enthalpy source dedicated to quantitative infrared emission spectroscopy of gas flows at elevated temperatures. Review of Scientific Instruments, 2019, 90 (9), pp.093103. 10.1063/1.5097696 . hal02309943

HAL Id: hal-02309943

https://hal-univ-rennes1.archives-ouvertes.fr/hal-02309943

Submitted on 28 Nov 2019

HAL is a multi-disciplinary open access archive for the deposit and dissemination of scientific research documents, whether they are published or not. The documents may come from teaching and research institutions in France or abroad, or from public or private research centers.
L'archive ouverte pluridisciplinaire HAL, est destinée au dépôt et à la diffusion de documents scientifiques de niveau recherche, publiés ou non, émanant des établissements d'enseignement et de recherche français ou étrangers, des laboratoires publics ou privés. 


\title{
High Enthalpy Source dedicated to quantitative infrared emission spectroscopy of gas flows at elevated temperatures
}

\section{R. Georges ${ }^{\mathrm{a}}$}

Univ Rennes, CNRS, IPR (Institut de Physique de Rennes) - UMR 6251, F-35000 Rennes, France

J. Thiévin,

Univ Rennes, CNRS, IPR (Institut de Physique de Rennes) - UMR 6251, F-35000 Rennes, France

A. Benidar

Univ Rennes, CNRS, IPR (Institut de Physique de Rennes) - UMR 6251, F-35000 Rennes, France

S. Carles

Univ Rennes, CNRS, IPR (Institut de Physique de Rennes) - UMR 6251, F-35000 Rennes, France

B. Amyay

Laboratoire Interdisciplinaire Carnot de Bourgogne (ICB), UMR 6303 CNRS, Université Bourgogne

Franche-Comté, 9 avenue A. Savary, BP 47870, 21078 DIJON Cedex, France

M. Louviot

Laboratoire Interdisciplinaire Carnot de Bourgogne (ICB), UMR 6303 CNRS, Université Bourgogne

Franche-Comté, 9 avenue A. Savary, BP 47870, 21078 DIJON Cedex, France

V. Boudon

Laboratoire Interdisciplinaire Carnot de Bourgogne (ICB), UMR 6303 CNRS, Université Bourgogne

Franche-Comté, 9 avenue A. Savary, BP 47870, 21078 DIJON Cedex, France

J. Vander Auwera

Service de Chimie Quantique et Photophysique, C.P. 160/09, Université Libre de Bruxelles, 50 avenue

F. D. Roosevelt, B-1050 Brussels, Belgium

\begin{abstract}
The High Enthalpy Source (HES) is a novel high temperature source developed to measure infrared line-by-line integrated absorption cross sections of flowing gases up to $2000 \mathrm{~K}$. The HES relies on a porous graphite furnace designed to uniformly heat a constant flow of gas. The flow compensates thermal dissociation by renewing continuously the gas sample and eliminating dissociation products. The flowing characteristics have been investigated using computational fluid dynamics simulation confirming good temperature uniformity. The HES has been coupled to a high-resolution Fourier transform spectrometer to record emission spectra of methane at temperatures ranging between 700 and $1400 \mathrm{~K}$. A radiative model has been developed to extract absolute line intensities from the recorded spectra.
\end{abstract}

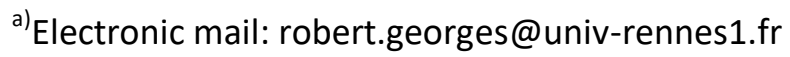




\section{INTRODUCTION}

Infrared spectroscopic data of high temperature gases (typically between 500 and $3000 \mathrm{~K}$ ) are essential for many areas of research. They are the bedrock of non-intrusive optical diagnostics of hot and harsh gaseous environments, such as flames ${ }^{1,2}$, plasmas ${ }^{3,4}$, scramjet or rocket exhausts, hypersonic shock layers, shock tubes ${ }^{5,6}$ or other industrial devices, for which the implementation of physical sensors and probes would be difficult or would perturb the measurement.

In hypersonic engineering, thermal transfers by radiation become preponderant at high temperature. Infrared spectroscopic data of relevant molecules are therefore of critical importance for evaluating the radiative energy losses of the shock layer forming in front of bodies flying at hypersonic velocities such as atmospheric reentry bodies (ballistic missile) or space probes ${ }^{7}$. In astrophysics, inversion of astronomical infrared signatures to retrieve concentration and temperature profiles of hot atmospheres (hot exoplanets, brown dwarfs, cooled carbon stars...), as well as the detection of new molecular species, relies on high-temperature spectroscopic data ${ }^{8,9}$. Last but not least, high temperature infrared data allows for the modelling of the internal dynamics (vibration, rotation, isomerization ${ }^{10}$ ) of polyatomic molecules, thus reinforcing our fundamental knowledge in the field of molecular physics.

The kind of information extracted from high-temperature spectroscopic data partly depends upon the instrumental resolution. Low-resolution experiments ${ }^{11-14}$ (typically $>1 \mathrm{~cm}^{-1}$ ) give access to the thermal radiance of whole vibrational bands while high-resolution is required to extract line-by-line parameters. The positions, ${ }^{1,3,15-19}$ integrated absorption cross sections ${ }^{20-24}$ (also called absolute line intensities) and shapes of lines can therefore be measured at high temperature. What makes hightemperature measurements especially crucial is their ability to give access to spectroscopic parameters associated with lines of high-J rotational quantum numbers, as well as with rotationvibration lines belonging to hot bands involving highly excited vibrational states, which are generally lacking in room temperature spectroscopic databases such as HITRAN ${ }^{25}$ and therefore cannot be extrapolated at high temperature. Lower state energies can be accessed using the so-called two temperature (2T) method ${ }^{19,20,26}$, provided that high-resolution spectra are recorded at least at two different temperatures. However, spectral congestion resulting from the overlapping of the enormous number of transitions characterizing high-temperature spectra clearly limits the line-byline approach. At very elevated temperatures, spectra of molecular species characterized by a large number of vibrational modes are composed of pseudo-resolved lines or "super lines" 27 , containing up to several dozens of blended transitions, sitting on top of a quasi-continuum formed by the accumulation of billions of weaker transitions originating from highly-excited vibrational energy levels (hot bands) ${ }^{22}$. The line-by-line experimental approach is also limited by the pressure broadening and line mixing effects, as is the case for example in shock tube experiments ${ }^{5,6}$ for which the pressure of the shocked gas sample exceeds the atmospheric pressure.

Various spectroscopic techniques have been implemented to probe high-temperature gases at lowor high- (Doppler limited) resolution. Among them, FTIR spectroscopy has been widely used to record either emission (see, e.g. refs. ${ }^{3,15,23,28}$ ) or absorption (see, e.g. refs. ${ }^{12,21,29}$ ) spectra. Tunable diode laser absorption spectroscopy is also a commonly-used technique to probe harsh gaseous media, as thoroughly described in several excellent review articles ${ }^{30,31}$. High-temperature data were also obtained by coupling a heated cell to intracavity laser absorption spectroscopy (ICLAS) ${ }^{32}$. More 
recently, an absorption spectrum of water vapor at $1950 \mathrm{~K}$ was recorded in a premixed methane/air flat flame using a cavity-enhanced optical frequency comb-based Fourier transform spectrometer ${ }^{2}$. Promising rapid-tuning external cavity quantum cascade laser based techniques are emerging to record broadband spectra in the mid-infrared, particularly suitable to probe shock-heated gases in a millisecond timescale ${ }^{6}$.

High-temperature line-by-line spectroscopic molecular parameters are generally compiled in dedicated databases such as HITEMP ${ }^{33}$, CDSD-4000 (carbon dioxide) ${ }^{34}$, MeCaSDa (methane) and ECaSDa (ethene) ${ }^{35}$. High-temperature experimental data is mandatory to validate molecular databases such as TheoRets ${ }^{36-38}$ and Exomol ${ }^{39,40}$ gathering purely ab initio calculated parameters.

High-temperature experimental devices face several problems. High-resolution absolute line intensity measurements or lower/medium-resolution band intensity measurements can be hindered by the presence of temperature gradients along the line of sight of either absorption $-{ }^{14}$ or emissionbased $^{20,26}$ experimental setups. Indeed, some high-temperature emitting cells inserted into tube furnaces are equipped with cooled windows leading to the formation of a zone of much lower gas temperature thereby re-absorbing emission lines associated with low rotational quantum numbers ${ }^{20,26}$. Several technical solutions were proposed to circumvent this drawback. Among them, the use of optical contacting sapphire windows, withstanding temperatures as high as $2273 \mathrm{~K}$ and not requiring cooling ${ }^{41}$, or laminar windows generating a counter-propagating gas flow to contain the test gas without physical windows ${ }^{12,42}$.

Furthermore, absorption experiments conducted at elevated temperatures have to take into account the thermal emission of the gas itself, which is superimposed to the absorption signal induced by the external source of photons. Successive recording of the absorption signal (external source activated) and the emission signal of the gas (external source deactivated) allows overcoming this problem $^{21,22,29}$ and notably increases the accuracy of the recorded cross sections, reaching a few percent $^{21}$.

Another effect impacting emission measurements arises from non-optically thin gas samples, which are prone to self-absorption effects. The intensity of the strong emission lines is more affected than the weak ones ${ }^{43}$ (see section IV).

Finally, to be reliable, line intensities deduced from emission measurements must be corrected from the transmission function of the setup, which includes the response of optical windows, mirrors, the detector, the optical filter, etc. Some Fourier transform infrared (FTIR) studies focus on the recording of narrow spectral ranges and line intensities are only corrected by the optical filter transmission curve $^{44}$ which is the main influencing optical element and which can be determined very accurately. In some cases ${ }^{23,26,43}$, a black body or a gray body source at the same temperature as the gaseous sample is used as a reference signal to correct the measured intensities from the spectral response function of the setup, thus achieving a better accuracy. On a different note, the very high temperature (more than $5000 \mathrm{~K}$ ) infrared emission FT spectral intensities produced using a plasma torch must be carefully corrected from the quartz or sapphire tube reflection, absorption and emission effects ${ }^{4}$.

This paper presents a device named "High Enthalpy Source" (HES) capable of reaching temperatures as high as $2000 \mathrm{~K}$ without relying on the use of optical windows. The HES device is coupled to a FT 
spectrometer (FTS) to produce high-resolution Doppler-limited emission spectra for quantitative spectroscopic fundamental studies. A method was developed to extract line-by-line absorption cross sections corrected from self-absorption effects and from the transmission function of the setup. This method is described hereafter and illustrated with results retrieved from high-temperature emission spectra of methane. Although less accurate and sensitive than other mature high temperature absorption ${ }^{22}$ or emission ${ }^{45}$ FTIR spectroscopic approaches, our high enthalpy source setup is presented as an alternative mean to measure absolute intensities in flowing conditions, particularly suitable for the investigation of species dissociating by pyrolysis.

\section{DESCRIPTION OF THE HIGH ENTHALPY SOURCE}

The HES prototype is shown in Fig. 1. The master piece of the device is a locally machined hollow porous graphite rod (PG-60; National Electrical Carbon Products, Inc.) open at one end. The inner diameter of the cavity is $8 \mathrm{~mm}$, while the wall thickness is $2 \mathrm{~mm}$. The function of the graphite rod is double. First, the cavity in the rod constitutes the gas furnace. With its small electric resistance (see Fig. 2) and high temperature resistance, graphite can be heated electrically up to very high temperatures, potentially much higher than $2000 \mathrm{~K}$. Second, due to the open porosity of graphite, the lateral wall of the rod behaves as a very efficient heat exchanger: a wall thickness of a few millimeters is enough to raise the temperature of the gas from room temperature up to $2000 \mathrm{~K}$ during its transit trough the wall. Furthermore, the continuous radial flow of gas entering the central cavity closed at one end ensures the absence of temperature gradients along the rod axis, as is evidenced by CFD two dimensional (2D) simulation (see Figs. 3 and 4). The electric power is provided by a stabilized $1 \mathrm{~kW}$ power supply. In typical operating conditions, an electrical current of a few tens of amperes and a few volts is delivered to the experimental device.

The carbon rod is maintained between two electrodes. The rear copper electrode, in contact with the closed end of the graphite rod, is spring mounted (not shown in Fig. 1) to absorb the thermal expansion of the graphite rod, which would break without this mechanical flexibility. The front electrode is made of non-porous isostatic carbon. An $8 \mathrm{~mm}$ diameter hole is made into this second electrode so that the radiation emitted by the gas can reach the spectrometer. The carbon electrode also plays the role of a nozzle evacuating the gas flow in a low-pressure discharge volume. The continuous renewal of the gas is necessary to compensate for its degradation over time. Small hydrocarbons such as acetylene or ethylene dissociate particularly quickly by pyrolysis at high temperatures so that static cells would lead to a continuous decrease of the emitted radiation over time. This is especially a problem with high-resolution FTIR recordings since they require hours to achieve a good signal to noise ratio. The carbon rod is inserted in a tantalum jacket. The incoming gas enters the annular space between the tantalum piece and the graphite rod through which it migrates under the action of a pressure difference ( $a$ few tens of $\mathrm{hPa}$ ) between the gas reservoir and the vacuum chamber, maintained by a series of roots blowers with adjustable pumping capacity. The tantalum jacket is surrounded by a water-cooled shield blocking the intense radiation it produces when brought to high temperature under the action of the graphite rod.

The emitted light is collected and transferred to a high-resolution FT spectrometer (Bruker IFS125HR) by a set of transfer optics. FTS and transfer optics are maintained under vacuum $\left(\sim 10^{-4} \mathrm{hPa}\right)$ to limit absorption by atmospheric water vapor and carbon dioxide. A window, made of either $\mathrm{CaF}_{2}$ or $\mathrm{ZnSe}$ 
according to the investigated spectral range, isolates the FTS from the vacuum chamber and a $2 \mathrm{~mm}$ diameter diaphragm pierced in a tantalum foil is located between the rod exit and the window (see Fig. 1). This diaphragm limits the amount of infrared radiation produced by the hot graphite reaching the detector and constitutes a gas baffle. A counter flow of dry nitrogen or dry argon is injected through the diaphragm to protect the window and to prevent stagnation of gas in the volume between the tantalum foil and the window.

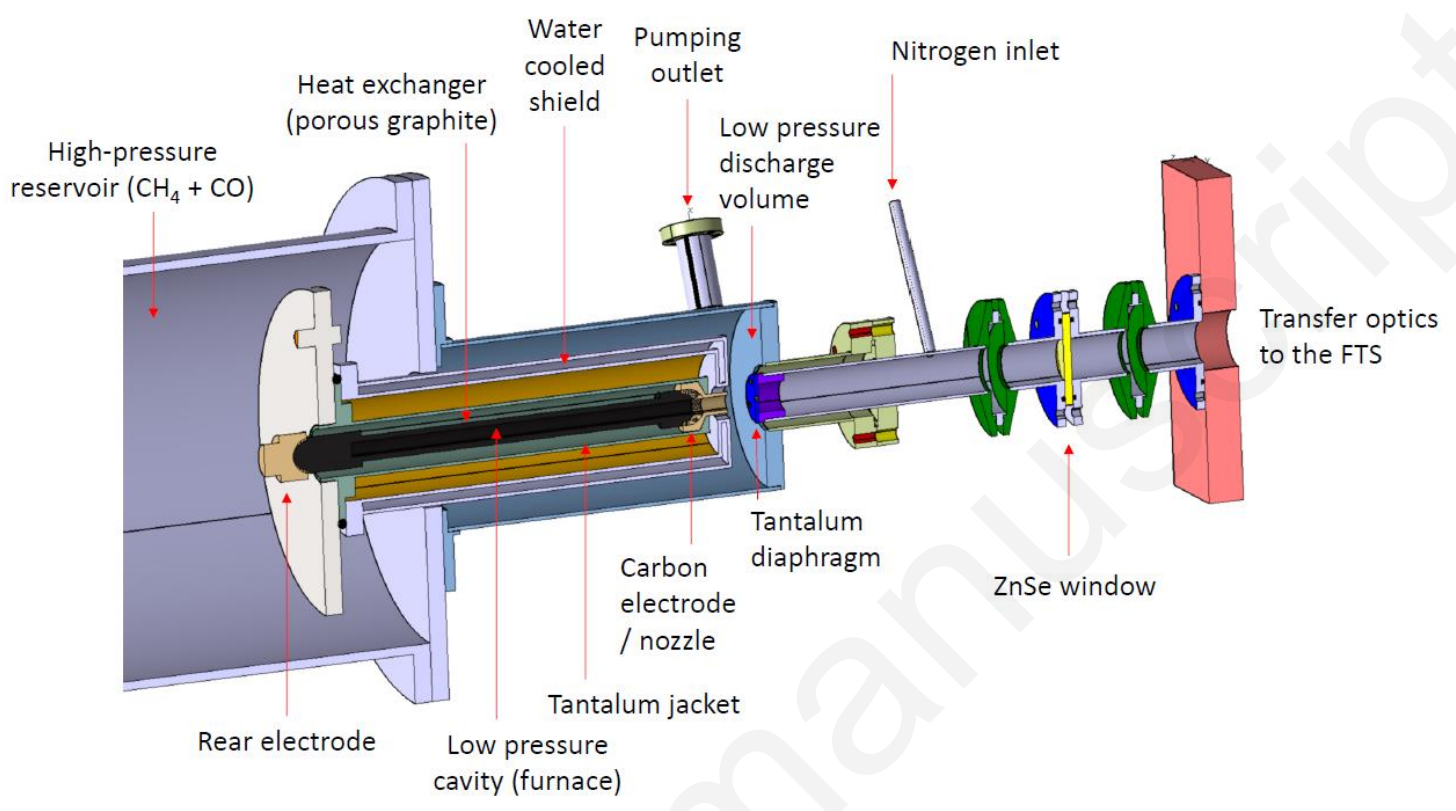

Fig. 1 - View of the High Enthalpy Source device. The total length of the graphite rod cavity is 21.6 $\mathrm{cm}$.

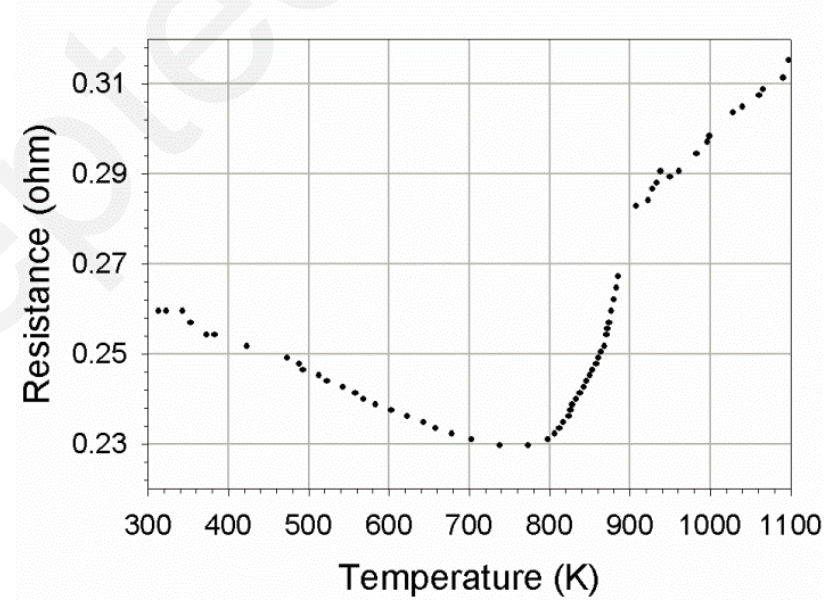

Fig. 2 - Evolution with temperature of the electrical resistance of the graphite rod. The resistance was determined using a 4-wire system. The successive decrease and increase of the resistance with temperature are typical of a semi-conductor behavior.

\section{CFD SIMULATION}


Computational Fluid Dynamic (CFD) 2D axi-symmetric simulation was performed with the ANSYS Fluent software to model the gas flow from the inlet of the graphite rod up to its ejection into the vacuum chamber (see Fig. 3). The flow of methane and the temperature of the furnace were set to 2 slm and $1400 \mathrm{~K}$, respectively. The flow field can be separated into two zones, respectively corresponding to the furnace cavity and the discharge chamber.

The temperature profile of the gas along the cavity axis is remarkably constant (see Fig. 4). Its uniform heating is a direct consequence of the radial inflow of the gas through the graphite walls and a key element of the HES device. This would not have been the case for a gas entering through one end of the hot cavity: a temperature gradient would inevitably be formed because of its progressive heating along the furnace ${ }^{46}$. This can be easily understood as follows. A gas is at the temperature of the walls after having traveled a certain distance called thermal entrance length $L_{e}$. For a circular tube of diameter $D$, the thermal entrance length is given by $L_{e} / D \approx 0.08 \operatorname{Re} \operatorname{Pr}$, where $\operatorname{Re}$ and $\operatorname{Pr}$ are the Reynolds number and the Prandtl number, respectively. The experimental conditions listed in Table 1 would lead to a thermal entrance length of several tens of centimeters.

The velocity of the gas revealed by the CFD simulation continuously increases from 0 at one end of the furnace cavity to a maximum value of about $400 \mathrm{~m} / \mathrm{s}$ at the other end. This latter velocity is not high enough to cause a significant Doppler frequency shift of the emission lines (calculated to be $0.004 \mathrm{~cm}^{-1}$ at $3000 \mathrm{~cm}^{-1}$ ). Additionally, the CFD simulation shows, from the rear electrode to the front nozzle electrode, a slight progressive increase of concentration followed by an abrupt drop close to the rod exit. The evolution of concentration along the axis of the furnace cavity is taken into account by introducing an effective molecular density into our 1D radiative model (see next section). The pressure gradient induces a progressive acceleration of the gas toward the front nozzle electrode from which it expands into the low pressure discharge chamber $(0.52-0.66 \mathrm{hPa}$, see Table I). This expansion is accompanied by a further, strong acceleration of the gas which reaches supersonic speeds. Such a supersonic regime shown by the CFD simulation is not surprising. Indeed, the ratio between the pressure in the chamber and the pressure in the hot cavity $(\sim 1 / 50)$ is sufficiently low to get a choked flow at the exit of the front electrode, which behaves actually like a sonic nozzle. As a result, a free-jet supersonic expansion naturally forms downstream the nozzle (the speed of sound is indeed reached by the gas at the nozzle exit as soon as the pressure ratio is smaller than $(2 /(\gamma+1))^{\gamma / \gamma-1}$, which is equal to 0.54 considering a specific heat ratio $\gamma$ of 1.32 for methane). As depicted in Figs. 3 and 4 , the supersonic gas expansion is accompanied by a drastic fall of temperature and concentration. The flow is further abruptly decelerated to subsonic speeds by a detached shock wave caused by the presence of the tantalum foil protecting the optical window. The shock wave is responsible for a sudden rise in temperature clearly visible in Fig. 4 . Figure 4 also shows that the gas density remains relatively low in the shock layer. As explained in the next section, the $1 \mathrm{D}$ radiative model shows that the presence of the shock wave has a negligible impact on the intensity of the infrared light emitted by the gas contained in the furnace cavity. 

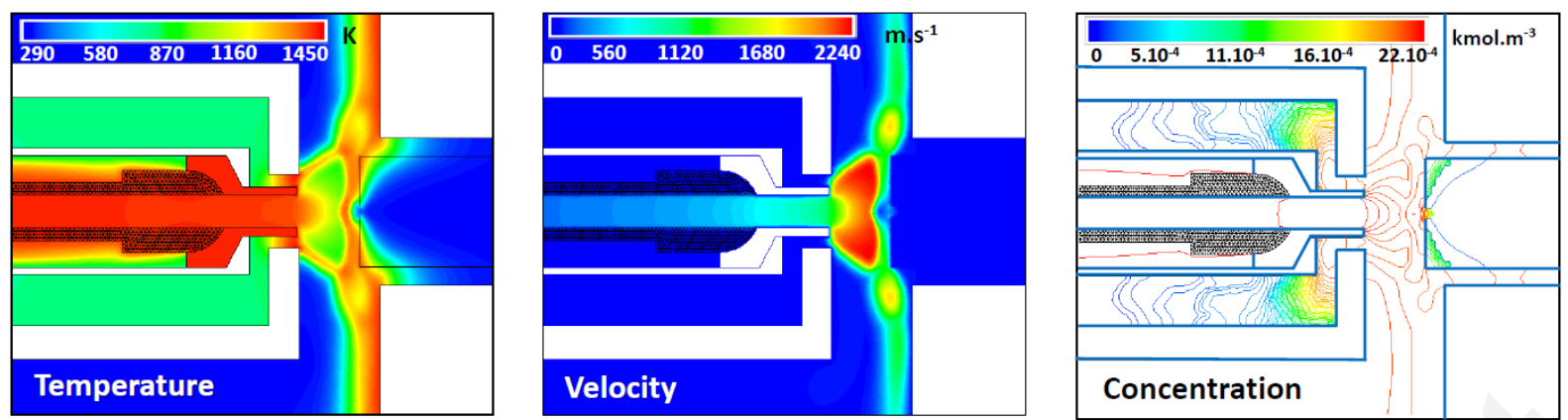

Fig. 3 - CFD simulation of a flow of methane $(2 \mathrm{slm})$ heated at $1400 \mathrm{~K}$. Left panel: temperature. Middle panel: velocity. Right panel: molecular concentration (molecular density). The gas is ejected at supersonic speed from the furnace and further decelerated by a detached shock wave forming in front of the tantalum foil protecting the optical window (not shown).
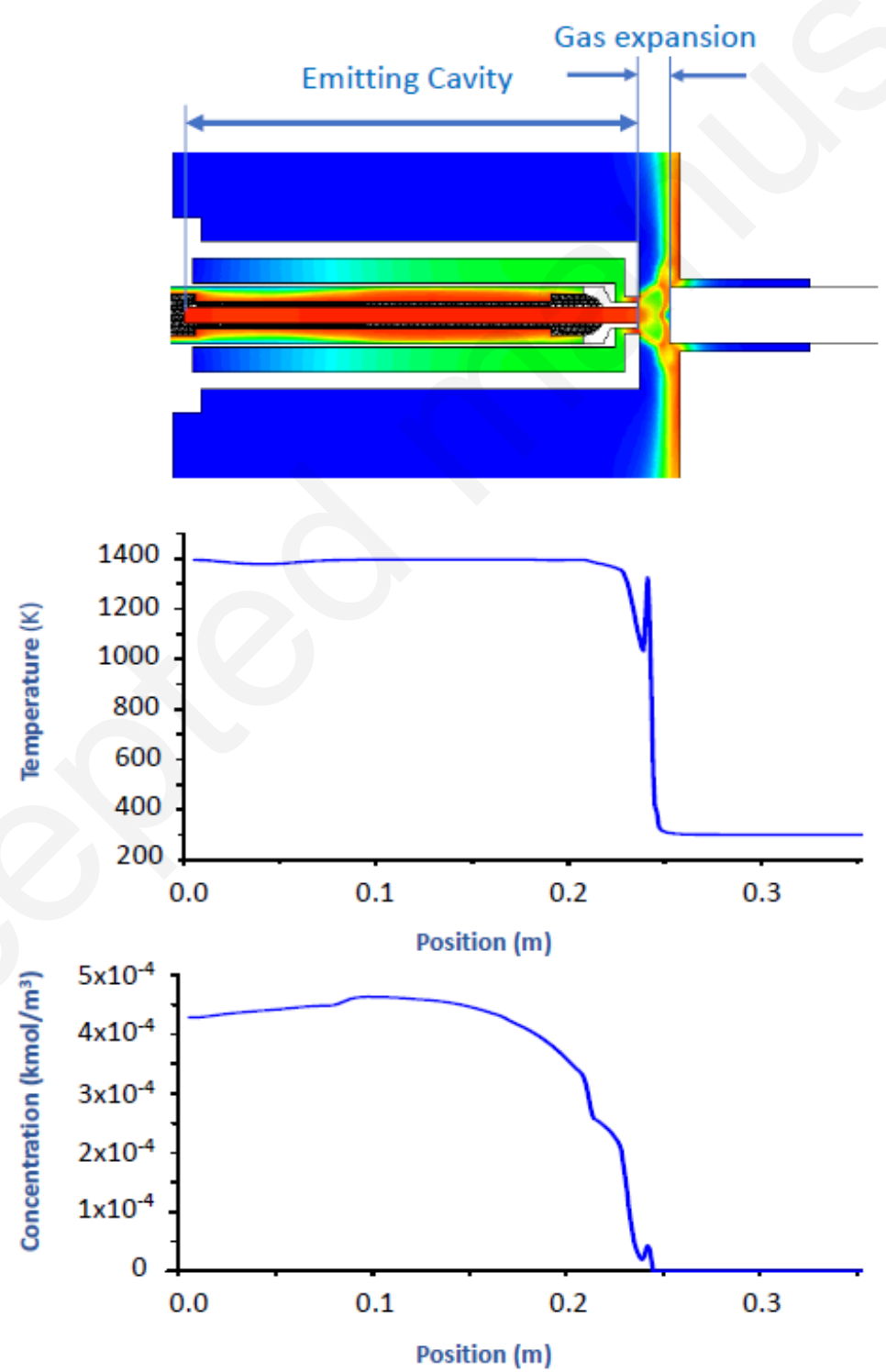

Fig. 4 - Upper panel: CFD simulation of the temperature of a flow $(2 \mathrm{~s} / \mathrm{m})$ of methane heated at $1400 \mathrm{~K}$. A supersonic expansion is formed in the discharge volume. The gas is further decelerated 
through the detached shock wave forming in front of the tantalum foil. Middle panel: CFD axial temperature profile from the hot cavity to the tantalum foil. Lower panel: density axial profile from the hot cavity to the tantalum foil.

\section{1D RADIATIVE TRANSFER MODEL}

The hot cavity is characterized by a spectral radiance $L_{\widetilde{v}}\left(T_{h o t}\right)$ composed of two terms, namely the thermal emission of the walls of the cavity, $L_{\widetilde{v}}^{\text {wall }}=\varepsilon L_{\widetilde{v}}^{\circ}\left(T_{\text {hot }}\right)$, partly absorbed by the molecules in the cavity (see upper panels of Fig. 5), and the molecular emission, which is partly self-absorbed (see middle panels of figure 5 ):

$$
\begin{gathered}
L_{\widetilde{v}}=\varepsilon L_{\widetilde{v}}^{\circ}\left(T_{h o t}\right) \tau_{h o t}+L_{\widetilde{v}}^{\circ}\left(T_{h o t}\right)\left(1-\tau_{h o t}\right) \\
L_{\widetilde{v}}=L_{\widetilde{v}}^{\circ}\left(T_{h o t}\right)\left[1-(1-\varepsilon) \tau_{h o t}\right]
\end{gathered}
$$

where $L_{\widetilde{v}}^{\circ}=2 h c^{2} \widetilde{v}^{3} /\left(\exp \left(\frac{h c \widetilde{v}}{k_{B} T_{h o t}}\right)-1\right)$ is the black body radiance $(\tilde{v}$ is the wavenumber, $c$ is the speed of light and $k_{B}$ is the Boltzmann constant); $\varepsilon$ is the graphite emissivity, which is slightly temperature dependent ${ }^{47} ; T_{\text {hot }}$ is the temperature of the cavity, and $\tau_{\text {hot }}=\exp (-\sigma(\tilde{v}) n \ell)$ is the transmittance of the cavity, with $\sigma(\tilde{v})$ the absorption cross section, $n$ the molecular density, and $\ell$ the length of the cavity.

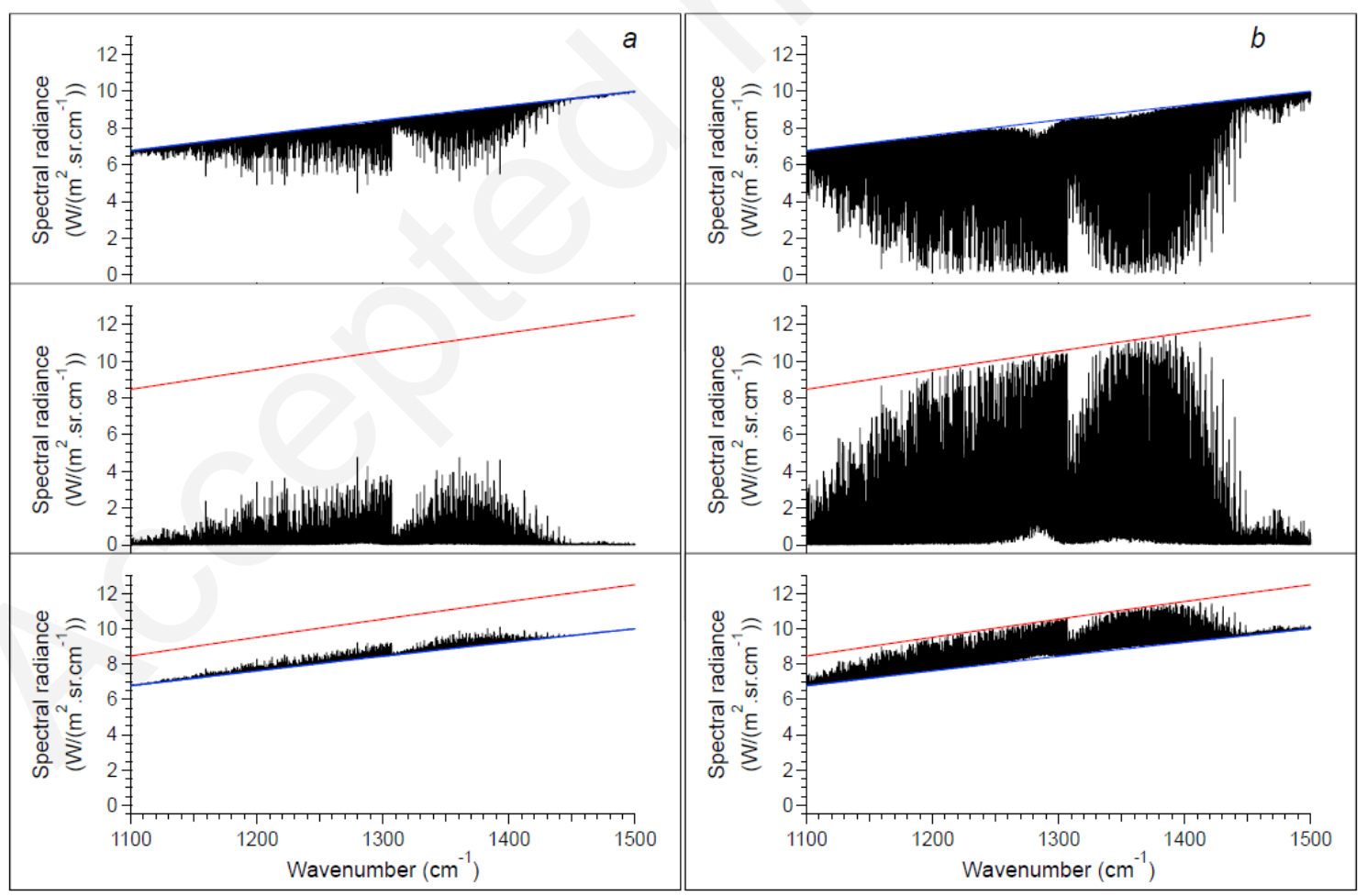

Fig. 5 - Contributions of the different terms included in the radiative model (Eq. 1), generated using the following conditions: $T_{\text {hot }}=1500 \mathrm{~K}, \ell=20.6 \mathrm{~cm}, \varepsilon=0.8$ and $n=10^{17}$ molecule $\mathrm{cm}^{-3}$ (panel $a$ ) or $n=$ $10^{18}$ molecule $\mathrm{cm}^{-3}$ (panel $b$ ). Methane lines were extracted from the MeCaSDa database ${ }^{35}$. Upper 
panels: wall emission absorbed by the hot gas; Middle panels: self-absorbed emission lines; Lower panels: spectral radiance at the exit of the emitting cavity. The gas emission appears between the graphite wall emission continuum (blue curve) and the blackbody emission curve (red curve).

The spectral radiance of the molecular lines, $L_{\widetilde{v}}^{\text {Lines }}\left(T_{\text {hot }}\right)$, can be obtained by subtracting the radiance of the walls from the total spectral radiance:

$$
L_{\widetilde{v}}^{\text {lines }}\left(T_{\text {hot }}\right)=L_{\widetilde{v}}-L_{\widetilde{v}}^{\text {wall }}\left(T_{\text {hot }}\right)=L_{\widetilde{v}}^{\circ}\left(T_{h o t}\right)(1-\varepsilon)\left(1-\tau_{\text {hot }}\right)
$$

The absorption cross section $\sigma_{k}(\tilde{v}, T)$ of the molecular line $k$ is related to its integrated absorption cross section $\bar{\sigma}_{k}(T)$ under the line profile $g\left(\tilde{v}-\tilde{v}_{k}\right)$ centered on the wavenumber $\tilde{v}_{k}$ :

$$
\sigma_{k}(\tilde{v}, T)=\bar{\sigma}_{k}(T) g\left(\tilde{v}-\tilde{v}_{k}\right)
$$

The integrated absorption cross section $\bar{\sigma}_{k}(T)$ can be calculated at any temperature from an absorption cross section $\bar{\sigma}_{k}\left(T_{0}\right)$ listed at a reference temperature $T_{0}(296 \mathrm{~K})$ in spectroscopic molecular databases (e.g. ref. ${ }^{25}$ ), using the following equation:

$$
\bar{\sigma}_{k}(T)=\bar{\sigma}_{k}\left(T_{0}\right) \frac{Q\left(T_{0}\right)}{Q(T)} \exp \left(\frac{E^{\prime \prime}}{k_{B} T_{0}}-\frac{E^{\prime \prime}}{k_{B} T}\right)\left[\frac{1-\exp \left(-\frac{\widetilde{v}_{k}}{k_{B} T}\right)}{1-\exp \left(-\frac{\widetilde{v}_{k}}{k_{B} T_{0}}\right)}\right]
$$

where $Q$ is the total partition function and $E^{\prime \prime}$ is the energy of the lower level of the transition corresponding to the line.

In Fig. 5 are modeled emission spectra corresponding to two different optical thicknesses, defined as $\sigma(\tilde{v}, T) n \ell$, differing by a factor 10 . It can be seen that a high optical thickness leads to a saturation of the strongest emission lines, their amplitude being limited by the black body emission curve (red curve in Fig. 5). It should be noted that this saturation effect is all the more important as the temperature is low because the partition function decreases with the temperature (see Eq. 4).

The absorption due to the cold expanding gas located between the cavity exit and the tantalum foil can be taken into account by introducing the transmittance $\tau_{\text {cold }}=\exp \left\{-\sigma_{\text {cold }}(\tilde{v}) n_{\text {cold }} \ell_{\text {cold }}\right\}$ of the cold gas layer in Eq. 1 . The spectral radiance at the aperture in the tantalum foil then becomes:

$$
L_{\widetilde{v}}=L_{\widetilde{v}}^{\circ}\left(T_{h o t}\right)\left[1-(1-\varepsilon) \tau_{h o t}\right] \tau_{c o l d}
$$

The modification of the radiance due to the absorbing contribution of the cold expanding gas was evaluated by coupling the 1D model (Eq. 1) to the CFD simulation. A too strong absorption is unwanted as it would notably affect emission line intensities. The CFD calculated gas concentration and temperature axial profiles are shown in Fig. 4 (lower and middle panels, respectively). They were obtained for a $2 \mathrm{~s} / \mathrm{m}$ flow of methane heated at $1400 \mathrm{~K}$. The CFD calculated grid domain was divided into 1-mm thick layers the respective radiances of which then summed using Eqs. 1 and 4 to generate the total radiance (i) at the exit of the gas furnace cavity (Fig. 6, upper panel) and (ii) after the gas expansion zone (Fig. 6, middle panel). The absorption due to the expanding zone was then obtained by subtracting these two radiances (Fig. 6, lower panel). It appears that the free-jet expansion only weakly absorbs light emitted by the hot cavity. Indeed, this absorption is at most $10 \%$ of the intensity of the strongest lines, while the weakest lines are marginally affected by the cold gas absorption. 
Such a low absorption provided by the present setup represents a significant improvement compared to other high temperature setup configurations in which the emission signal is strongly affected by the presence of cold gas layers (see, for example, the pioneering high-temperature highresolution emission studies of Nassar and Bernath ${ }^{28}$ or Hargreaves et al. $\left.{ }^{26}\right)$. The HES setup has therefore an interesting potential to measure the absolute intensity of high temperature emission lines, according to the procedure described hereafter.

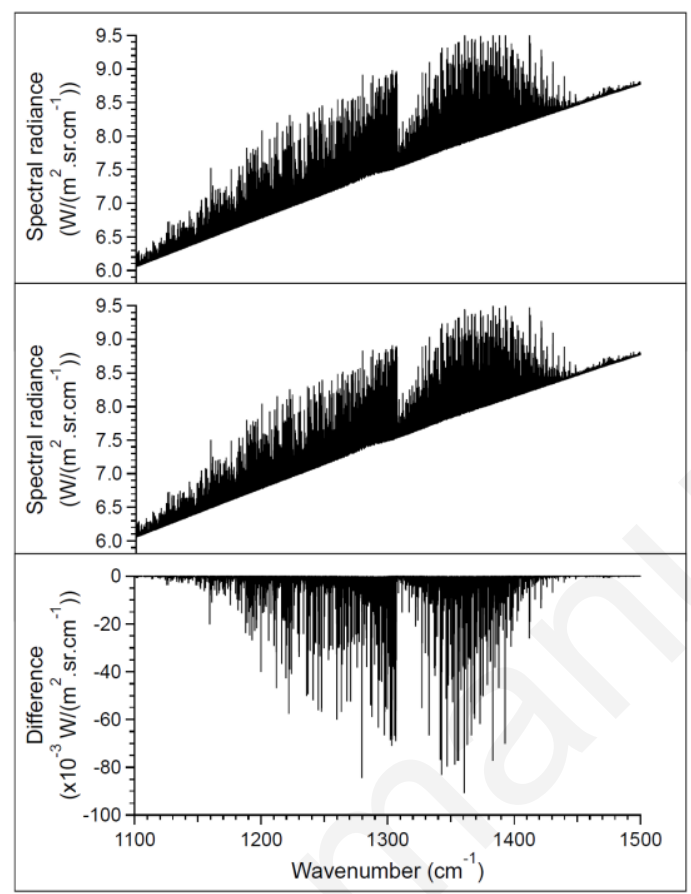

Fig.6 - Radiance relative to $2 \mathrm{~s} / \mathrm{m}$ of methane at $1400 \mathrm{~K}$. Upper panel: emitted radiance at the furnace exit. Middle panel: emitted radiance partly absorbed by the cooler gas expanding in the discharge volume. Lower panel: difference between the two previous contributions leading to the absorption contribution of the gas expanding in the discharge volume.

\section{FTS TRANSMAISSION FUNCTION}

Unlike absorption-based spectroscopic studies in which the transmission function of the spectrometer does not need to be considered in the analysis, emission spectroscopic studies must take it into account. Indeed, as mentioned in other studies ${ }^{23,44,48}$, each optical element making up the FTS (mirrors, detector, window, beamsplitter, optical filter) is characterized by its own transmission or reflection function, making it very difficult to derive the experimental transmission function of the setup. Hopefully, graphite can be considered a gray body characterized by a nearly constant emissivity in the $6.7-9 \mu \mathrm{m}$ spectral range considered in the present work ${ }^{47}$. However, it has been shown that the normal emissivity of graphite depends both on the surface roughness and the temperature ${ }^{47}$. Therefore, we decided to fit the emissivity for each investigated temperature condition.

The FTS transmission function can be straightforwardly estimated from the ratio of the continuum flux received by the detector, $\phi_{\widetilde{v}}^{\text {wall }}$, to the flux emitted by the walls and received by the detector, 
$\phi_{\widetilde{v}}^{\text {calc }}=F S \times \pi \varepsilon L_{\widetilde{v}}^{\circ}\left(T_{h o t}\right)$, calculated considering that they act as a gray body at the temperature $T_{\text {hot }}$ retrieved using the procedure described below. In this last equation, $\pi \varepsilon L_{\widetilde{v}}^{\circ}\left(T_{h o t}\right)$ is the spectral radiant flux emitted by unit area of the diaphragm pierced in the tantalum foil, assuming that the diaphragm of area $S$ acts as a Lambertian source. $F$ is a shape factor equal to the fraction of flux emitted by the diaphragm and intercepted by the collecting mirror. It is noteworthy that both the shape factor and the diaphragm area do not have to be explicitly determined as they do not depend on the temperature nor on the wavenumber. They can therefore be included in the FTS transmission function $f_{\widetilde{v}}^{\text {trans }}$ so that it can be written as:

$$
f_{\widetilde{v}}^{\text {trans }}\left(T_{\text {hot }}\right)=\frac{\phi_{\widetilde{v}}^{\text {wall }}}{L_{\widetilde{v}}^{\text {wall }}\left(T_{h o t}\right)}=\frac{\phi_{\widetilde{v}}^{\text {wall }}}{\varepsilon L_{\widetilde{v}}^{\circ}\left(T_{h o t}\right)}
$$

Because $f_{\widetilde{v}}^{\text {trans }}$ is temperature-dependent, the determination of the temperature $T_{\text {hot }}$ using the emission spectrum of carbon monoxide (see here below) involved an iterative procedure.

\section{ABSORPTION CROSS SECTION MEASUREMENTS}

\section{Rotational temperature of the hot gas}

The implementation of a thermocouple in the emitting cavity is not trivial because of the high intensity current circulating through the graphite rod. In the present work, the temperature was therefore retrieved using the intensities of emission lines of carbon monoxide admixed to the sample. To avoid self-absorption, care was taken to limit the injected amount of CO to $0.1 \mathrm{slm}$, the drawback being a decrease of the signal-to-noise ratio and thus an increase of the uncertainty on the measured temperatures (see Table 1).

The rotational temperature was extracted with the Boltzmann plot method using the following equation:

$$
\ln \left(\frac{\phi_{k}}{\widetilde{v}_{k}^{4} \times H L}\right)=-\frac{h c E^{\prime}}{k_{B} T_{h o t}}+C
$$

where $\phi_{k}=\int_{\text {line }} L_{\widetilde{v}}^{\text {lines }} \times f_{\widetilde{v}}^{\text {trans }} d \tilde{v}$ is the observed integrated flux of line $k$ associated with the molecular transition centered on the wavenumber $\tilde{v}_{k}$ ( $L_{\tilde{v}}^{\text {lines }}$ is defined in Eq. 2 and $\int_{\text {line }} d \tilde{v}$ means that the integration is made over a small spectral interval where only one line $\mathrm{k}$ is observed), $H L$ is the Hönl-London factor (for a diatomic molecule, $H L=J_{i}$ for P-branch lines and $H L=J_{i}+1$ for R-branch lines, including the rotational degeneracy of the lower level), $E^{\prime}$ is the energy of the upper level of the transition taken from HITRAN ${ }^{25}$ and $C$ is a constant.

\section{Extraction of emission line intensities}

Including the contribution of the FTS transmission function in Eq. 2, the observed spectral flux of the molecular lines is given by:

$$
\phi_{\widetilde{v}}^{\text {lines }}\left(T_{\text {hot }}\right)=\left[L_{\widetilde{v}}^{\circ}\left(T_{h o t}\right)(1-\varepsilon)\left(1-\exp \left\{-n \ell \sum_{k} \bar{\sigma}_{k}\left(T_{\text {hot }}\right) g\left(\tilde{v}-\tilde{v}_{k}\right)\right\}\right) \times f_{\widetilde{v}}^{\text {trans }}\left(T_{\text {hot }}\right)\right] * f_{\text {ils }}
$$


Equation 8 also includes the contribution of the instrument line shape function of the FTS, $f_{i l s}$ (* represents the convolution). In this work, $f_{i l s}$ included the effects of the finite maximum optical path difference and of the finite size of the entrance aperture of the interferometer. Thus, Eq. 8 directly links the recorded spectral flux $\phi_{\widetilde{v}}^{\text {lines }}\left(T_{\text {hot }}\right)$ of the methane emission lines corresponding to their absorption cross sections $\sigma_{k}\left(\tilde{v}, T_{h o t}\right)$, from which the integrated absorption cross section $\bar{\sigma}_{k}\left(T_{h o t}\right)$ can be easily determined using Eq. 3. Conversely, Eq. 8 can also be used to retrieve the molecular density of the emitting gas sample from known integrated absorption cross sections.

Equation 8 has been applied to the determination of the integrated absorption cross sections of methane lines at temperatures ranging from 700 to $1400 \mathrm{~K}$, relying on spectra recorded with the FTS fitted with a $\mathrm{HgCdTe}$ liquid nitrogen-cooled infrared detector, a $\mathrm{KBr}$ beamsplitter and an instrumental resolution set to $0.01 \mathrm{~cm}^{-1}$ (see Table I and Fig. 7). As Fig. 8 shows, the broadband spectral flux of the graphite rod was measured and removed ${ }^{49}$ prior to the analysis of the spectra. The model spectral radiance of Eq. 8 was fitted to the observed spectral radiance using a Levenberg-Marquardt nonlinear least squares fitting algorithm, giving each emission line a Gaussian line shape. The parameters determined for each spectrum were the density of methane ( $n$ in Eq. 8), the graphite emissivity and the intensities $\bar{\sigma}_{k}$ of methane lines.

The fitting procedure is composed of two steps which were iterated up to reach a convergence. Emission lines of the "Dyad - Ground State" cold bands and of the "Pentad - Dyad" hot bands were first identified using the MeCaSDA ${ }^{35}$ database, partly built on high-temperature spectroscopic studies performed using our High Enthalpy Source ${ }^{50,51}$. Then, in the first step, the most intense lines $\left(\geq 1 \times 10^{-}\right.$ ${ }^{22} \mathrm{~cm}^{-1} /\left(\right.$ molecule. $\left.\left.\mathrm{cm}^{-2}\right)\right)$ were selected and their absorption cross sections $\sigma_{k}\left(\tilde{v}, T_{\text {hot }}\right)$ were fixed (initially to their calculated value using Eq. (4)) in order to determine the gas density and the graphite emissivity. In the second step, the opposite has been done, the quantities $\varepsilon$ and $n$ were fixed while $\sigma_{k}\left(\tilde{v}, T_{\text {hot }}\right)$ were fitted.

The determined methane densities and emissivities are reported in Table I. The good matching between observed and calculated spectra, displayed in Fig. 8 for two different temperatures (Exp. 1 and 4 in Table I), highlights the reliability of our simple 1D radiative model. Note that, as the molecular density was revealed to be non-uniform along the furnace cavity by the CFD simulation (see Fig. 4), the molecular densities listed in Table I have to be considered as effective, which explains why their uncertainty is not provided.

Table I. Experimental conditions used to record emission spectra of methane

\begin{tabular}{|c|c|c|c|c|c|c|c|c|c|}
\hline Exp. & $\begin{array}{c}\text { Intensity } \\
\text { (A) }\end{array}$ & $\begin{array}{l}\text { Voltage } \\
\text { (V) }\end{array}$ & $\begin{array}{l}\text { Flow of } \\
\mathrm{CH}_{4}(\mathrm{slm})\end{array}$ & $\begin{array}{l}\text { Stagnation } \\
\text { pressure } \\
(\mathrm{hPa})\end{array}$ & $\begin{array}{l}\text { Discharge } \\
\text { chamber } \\
\text { pressure } \\
(\mathrm{hPa})\end{array}$ & $\begin{array}{c}\text { Gas sample } \\
\text { temperature } \\
\text { (K) }\end{array}$ & $\begin{array}{c}\text { Gas sample } \\
\text { molecular density } \\
\left(\text { molecule } \times \mathrm{cm}^{-3}\right)\end{array}$ & $\begin{array}{l}\text { Graphite } \\
\text { emissivity }\end{array}$ & $\begin{array}{l}\text { Correction } \\
\text { factor }\end{array}$ \\
\hline 1 & $20 \pm 0.1$ & $6.3 \pm 0.1$ & $4 \pm 0.03$ & $37.3 \pm 0.1$ & $0.66 \pm 0.002$ & $702 \pm 32$ & $1.56 \times 10^{17}$ & $0.88 \pm 0.05$ & 0.72 \\
\hline 2 & $30 \pm 0.1$ & $7.9 \pm 0.1$ & $4 \pm 0.03$ & $43.3 \pm 0.1$ & $0.66 \pm 0.002$ & $886 \pm 24$ & $1.07 \times 10^{17}$ & $0.82 \pm 0.05$ & 0.83 \\
\hline 3 & $50 \pm 0.1$ & $11.9 \pm 0.1$ & $4 \pm 0.03$ & $50.0 \pm 0.1$ & $0.66 \pm 0.002$ & $1173 \pm 26$ & $6.3 \times 10^{16}$ & $0.77 \pm 0.10$ & 0.87 \\
\hline 4 & $50 \pm 0.1$ & $11.5 \pm 0.1$ & $2 \pm 0.02$ & $33.5 \pm 0.1$ & $0.52 \pm 0.002$ & $1246 \pm 25$ & $4.0 \times 10^{16}$ & $0.77 \pm 0.10$ & 0.80 \\
\hline 5 & $70 \pm 0.1$ & $15.0 \pm 0.1$ & $4 \pm 0.03$ & $59.3 \pm 0.1$ & $0.66 \pm 0.002$ & $1401 \pm 21$ & $6.2 \times 10^{16}$ & $0.80 \pm 0.10$ & 0.81 \\
\hline
\end{tabular}




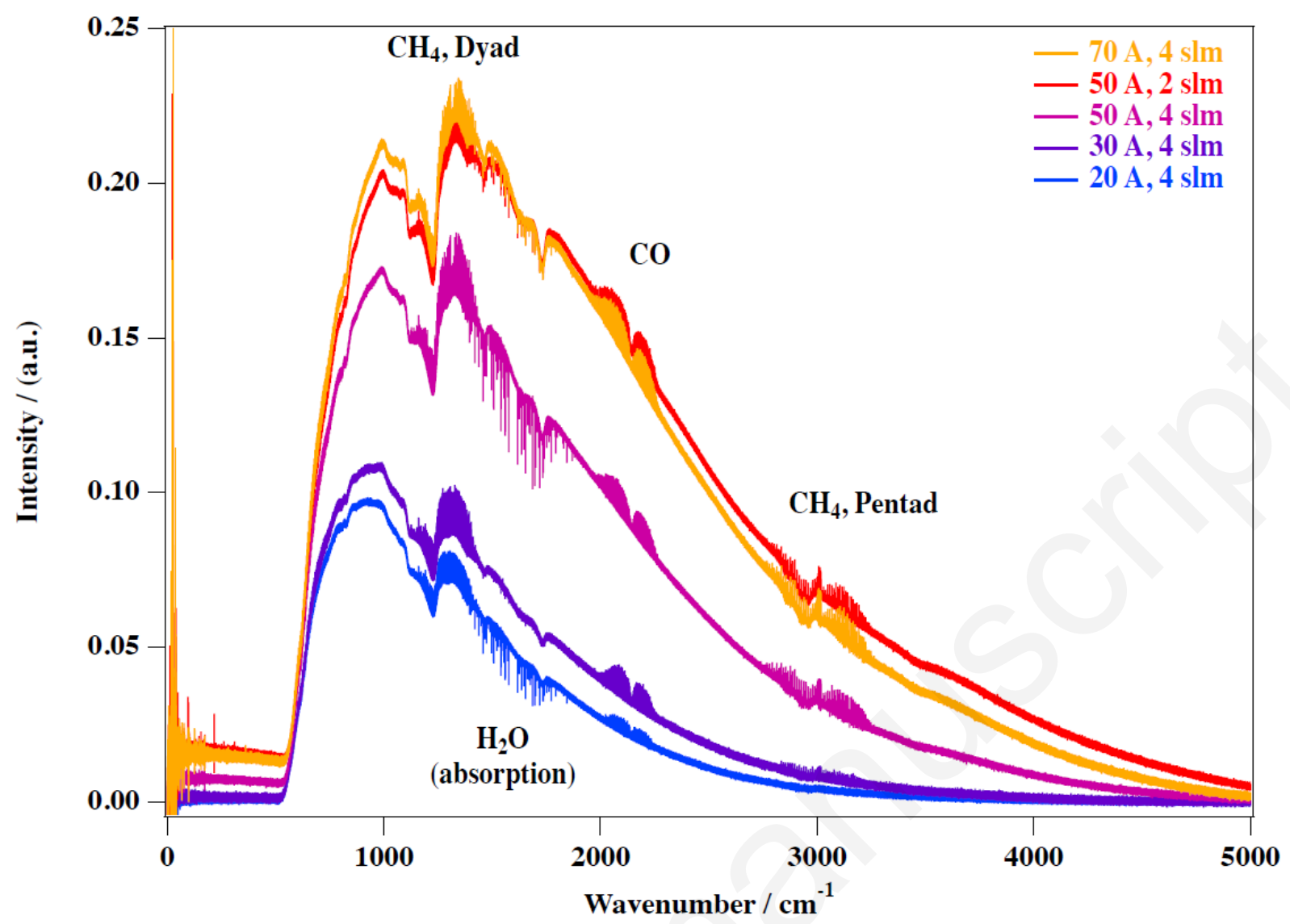

Fig. 7 - Raw emission FT spectra of methane recorded in this work. The dyad and pentad emission bands of methane are centered around 1300 and $3000 \mathrm{~cm}^{-1}$, respectively. The 1-0 emission band of carbon monoxide is centered around $2150 \mathrm{~cm}^{-1}$ and absorption due to atmospheric water is observed around $1700 \mathrm{~cm}^{-1}$. The temperature increase is associated with an increase of the spectral radiance of the graphite rod.
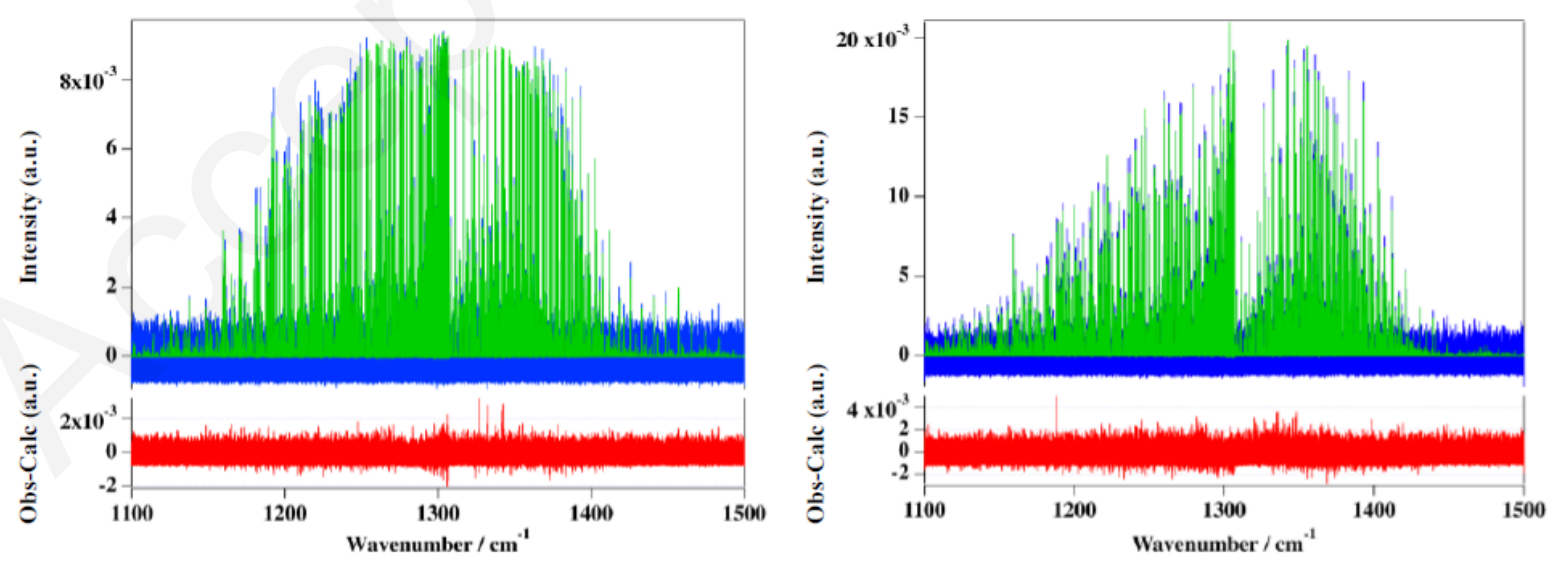

Fig. 8 - Raw emission FT spectra of methane at $702 \pm 32 \mathrm{~K}$ (left) and $1246 \pm 25 \mathrm{~K}$ (right) displayed in Fig.7, from which the continuum emission has been subtracted. Blue curves: experimental data. Green curves: best-fit calculated spectra. Red curves: differences between the observed and calculated spectra. 


\section{Comparison with other experimental data}

Figure 9 compares line intensities of methane measured the highest temperature of $1401 \mathrm{~K}$ in the present work (Exp. 5 in Table 1) and extracted from the 2016 edition of the HITRAN molecular database $^{25}$, extrapolated at the experimental temperature using Eq. 4. It shows that the line intensities obtained at $1401 \mathrm{~K}$ are systematically overestimated. Similar overestimations are observed for the other experiments conducted in this work. A correction factor (i.e. the inverse of the slope of a line fitted to the data shown in Fig. 9) between 0.72 and 0.87 depending on the experimental conditions (see last column of Table I) was therefore applied to match the HITRAN intensities.

The discrepancies observed are attributed to a strong correlation between the integrated cross sections $\bar{\sigma}_{k}$ of the methane lines, the emissivity, and the effective molecular density $n$, which are extracted during the same fitting procedure. The effect of such a correlation can be understood in the case of small $\bar{\sigma}_{k}$ and/or $n$ values. In that case, Eq. 8 reduces to the linear relation $L_{\widetilde{v}}^{\text {lines }}\left(T_{\text {hot }}\right) \approx$ $L_{\widetilde{v}}^{\circ}\left(T_{h o t}\right)(1-\varepsilon)\left[n \ell \sum_{k} \bar{\sigma}_{k}\left(T_{h o t}\right) g\left(\tilde{v}-\tilde{v}_{k}\right)\right] \times f_{\widetilde{v}}^{\text {trans }}\left(T_{h o t}\right)$ for which several couples of $\left[n, \bar{\sigma}_{k}\left(T_{h o t}\right)\right]$ values lead to the same $\bar{\sigma}_{k}\left(T_{h o t}\right) n \ell$ product. This correlation could be eliminated by determining the density independently. With this aim in view, a direct gas sample temperature measurement is under study that, coupled to the measured gas sample pressure, should lead to the molecular density through the ideal gas law $P=n k T$.

An alternative explanation of such a deviation comes from the fact that at high temperature, the lines observed are obviously not "individual lines", but rather "super lines ${ }^{27 "}$ made up of multiple components. Extracted intensities are therefore probably systematically overestimated with respect to intensities extrapolated at high temperature from the HITRAN database.

It is important to emphasize however that, for a given temperature, one correction factor applies to the whole ensemble of measured line intensities $\bar{\sigma}_{k}$. The approach used in the present work therefore remains an efficient method to measure, or at least to estimate with a reasonable precision, the absolute intensities of unidentified molecular transitions, in particular those corresponding to hot bands the contribution of which becoming very significant at high temperature, as well as those corresponding to high-J values. Once calibrated, the uncertainty on the line intensities $\bar{\sigma}_{k}$ was estimated to be a few percent, reaching up to $10 \%$ for the strongest lines that are affected the most by the absorption of the expanding gas in the discharge volume as discussed in section IV. 

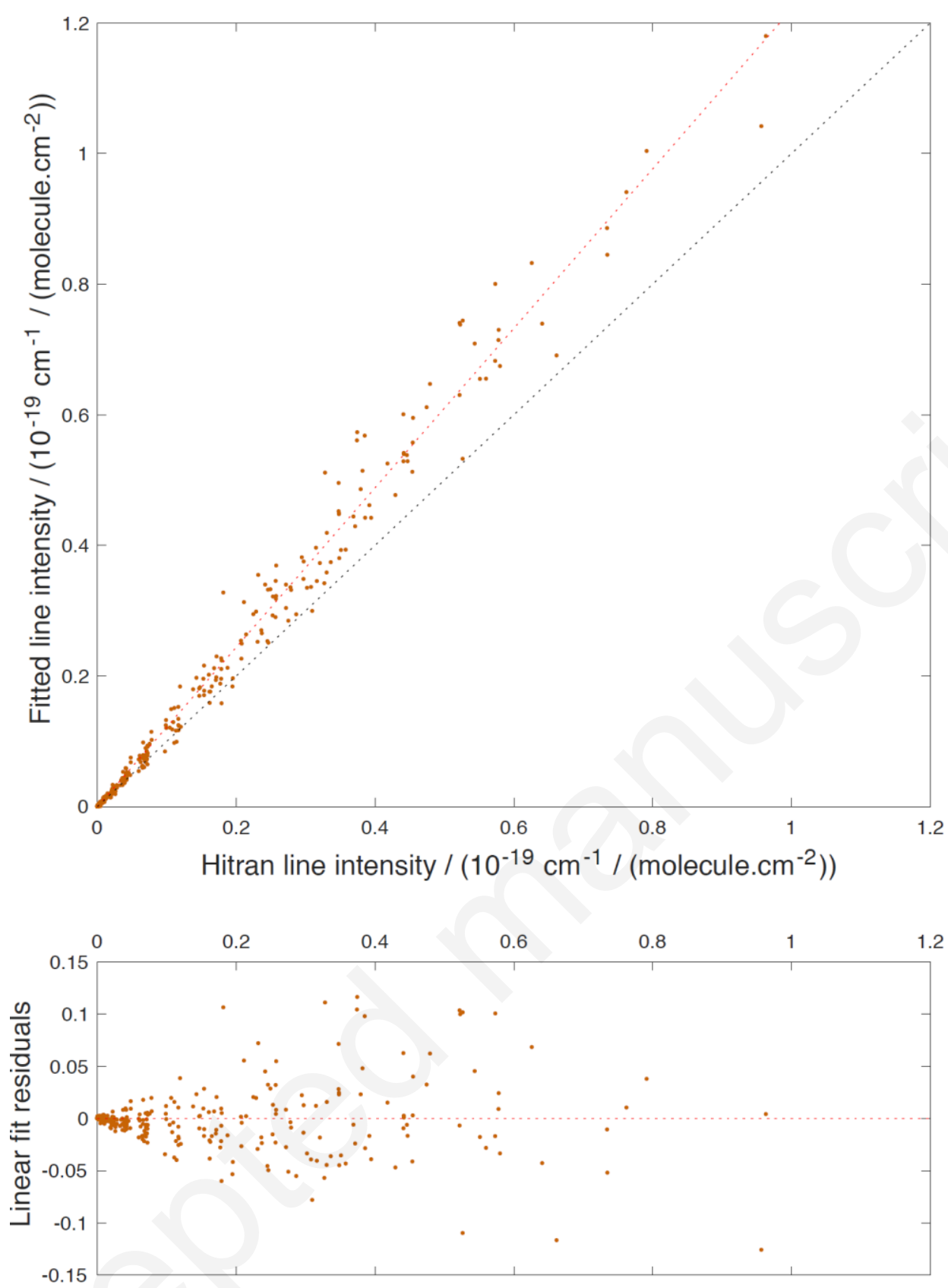

Fig. 9 - Upper panel: Comparison between line intensities measured in the present work (spectrum recorded at $1401 \mathrm{~K}$; Exp. 5 in Table 1) and available in the HITRAN database ${ }^{24}$, extrapolated to $1401 \mathrm{~K}$. The dashed line of slope one is drawn to ease the comparison. The fitted line intensities are overestimated by a factor of about $20 \%$ (see text). Lower panel: linear fit residuals.

\section{Conclusion}

The high enthalpy source is a novel type of compact high temperature source that enables heating a continuous flow of gas up to $2000 \mathrm{~K}$ from which absolute line intensities can be extracted. As the flow ensures a constant renewal of the gaseous sample, this system is of particular relevance for the spectroscopic study of molecular species dissociating at high temperature. The cylindrical furnace made of porous graphite was designed to be filled radially to maintain a uniform temperature within 
the flowing gas sample. The hydrodynamic characteristics of the flow were investigated using computational fluid dynamic simulation confirming the good uniformity of the temperature. The high enthalpy source was connected to a high-resolution Fourier transform spectrometer to analyze the infrared radiation emitted by methane heated up to temperatures between 700 and $1400 \mathrm{~K}$. Two successive steps were applied to extract absolute line intensities from the emission spectra. In a first step, emission spectra were corrected for the transmission function of the spectrometer, assuming that graphite radiates as a gray body in the infrared. Although complex, this spectral response function could be readily determined from the continuous emission baseline originating from the graphite furnace walls.

A method, based on a simple 1D radiative transfer model, was then developed to directly extract the integrated absorption cross sections of methane lines from the recorded emission spectra. However, a strong correlation between molecular densities and integrated absorption cross sections was responsible for an overestimation of about $20-30 \%$ of the measured line intensities compared to information available in HITRAN 2016. Therefore, in a second step, a calibration factor derived from the comparison with line intensities available in HITRAN 2016 was applied to correct the measured line intensities, leading to an uncertainty to about $10 \%$.

In the near future, the temperature will be determined independently using an optical pyrometer. Coupled to the measurement of the gas pressure, the gas density will be determined independently from the line intensities of the gaseous sample. The present setup will therefore provide an alternative to absorption spectroscopy methods for determining the high temperature line-by-line infrared absorption cross sections of high temperature gases in flowing conditions.

\section{ACKNOWLEDGEMENTS}

This work is supported by the French National Research agency (ANR) in the frame of the e-PYTHEAS project. J.V.A. acknowledges financial support from the "Fonds de la Recherche Scientifique" F.R.S.FNRS (Belgium, Contract No. CDR J.0123.16). R.G. acknowledges the AILES beamline manager Pascale Roy for providing access to the high-resolution FTS available on the beamline (project 99120128) and O. Pirali for his help with the recordings.

\section{REFERENCES}

${ }^{1}$ D. Bailly, C. Camy-Peyret, and R. Lanquetin, Journal of Molecular Spectroscopy 182, 10 (1997).

${ }^{2}$ L. Rutkowski, A. Foltynowicz, F.M. Schmidt, A.C. Johansson, A. Khodabakhsh, A.A. Kyuberis, N.F. Zobov, O.L. Polyansky, S.N. Yurchenko, and J. Tennyson, Journal of Quantitative Spectroscopy and Radiative Transfer 205, 213 (2018).

${ }^{3}$ D. Bailly, O. Pirali, and M. Vervloet, Journal of Molecular Spectroscopy 222, 180 (2003).

${ }^{4}$ S. Depraz, M.Y. Perrin, and A. Soufiani, Journal of Quantitative Spectroscopy and Radiative Transfer 113, 1 (2012).

${ }^{5}$ I. Stranic and R.K. Hanson, Journal of Quantitative Spectroscopy and Radiative Transfer 142, 58 (2014).

${ }^{6}$ C.L. Strand, Y. Ding, S.E. Johnson, and R.K. Hanson, Journal of Quantitative Spectroscopy and Radiative Transfer 222-223, 122 (2019). 
${ }^{7}$ Cardone, Gennaro, Quantitative InfraRed Thermography Journal 4, 233 (2007).

${ }^{8}$ G. Tinetti, T. Encrenaz, and A. Coustenis, Astron Astrophys Rev 21, 63 (2013).

${ }^{9}$ T. Feng, H. Xiong, and W. Ji, Chinese Astronomy and Astrophysics 41, 530 (2017).

${ }^{10}$ G.Ch. Mellau, J. Chem. Phys. 133, 164303 (2010).

${ }^{11}$ S. Clausen and J. Bak, Meas. Sci. Technol. 13, 1223 (2002).

${ }^{12}$ H. Grosch, A. Fateev, K.L. Nielsen, and S. Clausen, Journal of Quantitative Spectroscopy and Radiative Transfer 130, 392 (2013).

${ }^{13}$ S.P. Bharadwaj and M.F. Modest, Journal of Quantitative Spectroscopy and Radiative Transfer 103, 146 (2007).

${ }^{14}$ K. Wakatsuki, S.P. Fuss, A. Hamins, and M.R. Nyden, Proceedings of the Combustion Institute 1, 1565 (2005).

${ }^{15}$ P.-F. Coheur, P.F. Bernath, M. Carleer, R. Colin, O.L. Polyansky, N.F. Zobov, S.V. Shirin, R.J. Barber, and J. Tennyson, J. Chem. Phys. 122, 074307 (2005).

${ }^{16}$ G.Ch. Mellau, J. Chem. Phys. 134, 194302 (2011).

${ }^{17}$ G.Ch. Mellau, Journal of Molecular Spectroscopy 264, 2 (2010).

${ }^{18}$ G.Ch. Mellau, Journal of Molecular Spectroscopy 269, 12 (2011).

${ }^{19}$ M. Ghysels, S. Vasilchenko, D. Mondelain, S. Béguier, S. Kassi, and A. Campargue, Journal of Quantitative Spectroscopy and Radiative Transfer 215, 59 (2018).

${ }^{20}$ R.J. Hargreaves, C.A. Beale, L. Michaux, M. Irfan, and P.F. Bernath, ApJ 757, 46 (2012).

${ }^{21}$ R.J. Hargreaves, E. Buzan, M. Dulick, and P.F. Bernath, Molecular Astrophysics 1, 20 (2015).

${ }^{22}$ R.J. Hargreaves, P.F. Bernath, J. Bailey, and M. Dulick, ApJ 813, 12 (2015).

${ }^{23}$ J. Thiévin, R. Georges, S. Carles, A. Benidar, B. Rowe, and J.-P. Champion, Journal of Quantitative Spectroscopy and Radiative Transfer 109, 2027 (2008).

${ }^{24}$ A.V. Nikitin, M. Rey, and V.G. Tyuterev, Journal of Quantitative Spectroscopy and Radiative Transfer 200, 90 (2017).

${ }^{25}$ I.E. Gordon, L.S. Rothman, C. Hill, R.V. Kochanov, Y. Tan, P.F. Bernath, M. Birk, V. Boudon, A. Campargue, K.V. Chance, B.J. Drouin, J.-M. Flaud, R.R. Gamache, J.T. Hodges, D. Jacquemart, V.I. Perevalov, A. Perrin, K.P. Shine, M.-A.H. Smith, J. Tennyson, G.C. Toon, H. Tran, V.G. Tyuterev, A. Barbe, A.G. Császár, V.M. Devi, T. Furtenbacher, J.J. Harrison, J.-M. Hartmann, A. Jolly, T.J. Johnson, T. Karman, I. Kleiner, A.A. Kyuberis, J. Loos, O.M. Lyulin, S.T. Massie, S.N. Mikhailenko, N. MoazzenAhmadi, H.S.P. Müller, O.V. Naumenko, A.V. Nikitin, O.L. Polyansky, M. Rey, M. Rotger, S.W. Sharpe, K. Sung, E. Starikova, S.A. Tashkun, J.V. Auwera, G. Wagner, J. Wilzewski, P. Wcisło, S. Yu, and E.J. Zak, Journal of Quantitative Spectroscopy and Radiative Transfer 203, 3 (2017).

${ }^{26}$ R.J. Hargreaves, G. Li, and P.F. Bernath, ApJ 735, 111 (2011).

${ }^{27}$ S.N. Yurchenko, D.S. Amundsen, J. Tennyson, and I.P. Waldmann, A\&A 605, A95 (2017).

${ }^{28}$ R. Nassar and P. Bernath, Journal of Quantitative Spectroscopy and Radiative Transfer 82, 279 (2003).

${ }^{29}$ M.F. Modest and S.P. Bharadwaj, Journal of Quantitative Spectroscopy and Radiative Transfer 73, 329 (2002).

${ }^{30}$ C.S. Goldenstein, R.M. Spearrin, Jay.B. Jeffries, and R.K. Hanson, Progress in Energy and Combustion Science 60, 132 (2017).

${ }^{31}$ M.A. Bolshov, Yu.A. Kuritsyn, and Yu.V. Romanovskii, Spectrochimica Acta Part B: Atomic Spectroscopy 106, 45 (2015).

${ }^{32}$ T. Petrova, Y. Poplavskii, V. Serdyukov, and L. Sinitsa, Molecular Physics 104, 2691 (2006).

${ }^{33}$ L.S. Rothman, I.E. Gordon, R.J. Barber, H. Dothe, R.R. Gamache, A. Goldman, V.I. Perevalov, S.A. Tashkun, and J. Tennyson, Journal of Quantitative Spectroscopy and Radiative Transfer 111, 2139 (2010).

${ }^{34}$ S.A. Tashkun and V.I. Perevalov, Journal of Quantitative Spectroscopy and Radiative Transfer 112, 1403 (2011).

${ }^{35}$ Y.A. Ba, C. Wenger, R. Surleau, V. Boudon, M. Rotger, L. Daumont, D.A. Bonhommeau, V.G. Tyuterev, and M.-L. Dubernet, Journal of Quantitative Spectroscopy and Radiative Transfer 130, 62 (2013). 
${ }^{36}$ M. Rey, A.V. Nikitin, B. Bézard, P. Rannou, A. Coustenis, and V.G. Tyuterev, Icarus 303, 114 (2018).

${ }^{37}$ M. Rey, A.V. Nikitin, and V.G. Tyuterev, ApJ 789, 2 (2014).

${ }^{38}$ M. Rey, A.V. Nikitin, Y.L. Babikov, and V.G. Tyuterev, Journal of Molecular Spectroscopy 327, 138 (2016).

${ }^{39}$ S.N. Yurchenko and J. Tennyson, Mon Not R Astron Soc 440, 1649 (2014).

${ }^{40}$ J. Tennyson, S.N. Yurchenko, A.F. Al-Refaie, E.J. Barton, K.L. Chubb, P.A. Coles, S. Diamantopoulou, M.N. Gorman, C. Hill, A.Z. Lam, L. Lodi, L.K. McKemmish, Y. Na, A. Owens, O.L. Polyansky, T. Rivlin, C. Sousa-Silva, D.S. Underwood, A. Yachmenev, and E. Zak, Journal of Molecular Spectroscopy 327, 73 (2016).

${ }^{41}$ S.T. Melin and S.T. Sanders, Journal of Quantitative Spectroscopy and Radiative Transfer 180, 184 (2016).

${ }^{42}$ S. Clausen and J. Bak, Journal of Quantitative Spectroscopy and Radiative Transfer 61, 131 (1999).

${ }^{43}$ A. Moudens, R. Georges, A. Benidar, B. Amyay, M. Herman, A. Fayt, and B. Plez, Journal of Quantitative Spectroscopy and Radiative Transfer 112, 540 (2011).

${ }^{44}$ G.Ch. Mellau, B.P. Winnewisser, and M. Winnewisser, Journal of Molecular Spectroscopy 249, 23 (2008). ${ }^{45}$ (n.d.).

${ }^{46}$ A.F. Mills, Heat Transfer, 2 edition (Prentice Hall, Upper Saddle River, N.J, 1998).

${ }^{47}$ F. Wang, L. Cheng, H. Mei, Q. Zhang, and L. Zhang, International Journal of Thermophysics 35, 62 (2014).

${ }^{48}$ J.P. Hofmann, B. Eifert, and G.Ch. Mellau, Journal of Molecular Spectroscopy 262, 75 (2010).

${ }^{49}$ M. Morháč, Nuclear Instruments and Methods in Physics Research Section A: Accelerators, Spectrometers, Detectors and Associated Equipment 600, 478 (2009).

${ }^{50}$ B. Amyay, M. Louviot, O. Pirali, R. Georges, J. Vander Auwera, and V. Boudon, The Journal of Chemical Physics 144, 024312 (2016).

${ }^{51}$ B. Amyay, A. Gardez, R. Georges, L. Biennier, J. Vander Auwera, C. Richard, and V. Boudon, The Journal of Chemical Physics 148, 134306 (2018). 